\title{
Glucagon for hypoglycaemia in infants small for gestational age
}

\author{
P E CARTER, D J LLOYD, AND P DUFFTY \\ Neonatal Unit, Aberdeen Maternity Hospital, and Department of Child Health, University of Aberdeen
}

SUMMARY Twenty five infants who were small for gestational age received glucagon $(0.5 \mathrm{mg} /$ day by continuous infusion) in the treatment of hypoglycaemia. Twenty responded within three hours with a rise in blood glucose concentration to above 4 $\mathrm{mmol} / \mathrm{l}$. Five subjects subsequently required hydrocortisone to maintain glucose concentrations. Rebound hypoglycaemia occurred in nine infants after rapid discontinuation of glucagon or interruption of the intravenous infusions. Response was poor after maternal $\beta$ blockade.

Hypoglycaemia is a common problem in the infant who is small for gestational age. Many paediatricians use corticosteroids if the hypoglycaemia fails to respond to high caloric intake. Although glucagon is often used for the management of hypoglycaemia in the infant of the diabetic mother, in a recent textbook of neonatology no reference is made to its use in infants who are small for gestational age. ${ }^{1} \mathrm{~A}$ recent paper did, however, describe low glucagon concentrations in a group of hypoglycaemic term infants, successfully treated with a single intravenous bolus of glucagon. ${ }^{2}$ For several years we have used a continuous glucagon infusion as the first line of treatment if hypoglycaemia persists despite high energy oral feeds and concentrated solutions of intravenous dextrose. We report here our experience over the last five years.

\section{Patients and methods}

Patients were all infants born at Aberdeen Maternity Hospital between January 1982 and December 1986, whose birth weight was below the 5th centile for gestational age, and in whom it had not been possible to maintain capillary blood glucose concentration above $2 \mathrm{mmol} / \mathrm{l}$ by the use of intravenous dextrose $12.5 \%$ solution at appropriate volumes (supplying $6.5 \mathrm{mg} / \mathrm{kg} /$ minute of glucose on day 1) and a bolus of dextrose $0.2 \mathrm{~g} / \mathrm{kg}$ together with frequent feeds of high energy milk (Prematalac, Cow and Gate).

Glucagon was infused continuously in $10 \%$ dextrose at a standard dosage of $0.5 \mathrm{mg} /$ day and increased as necessary to maintain capillary blood glucose concentration at or above $4 \mathrm{mmol} / \mathrm{l}$ (BM
Test, Glycaemie) as in our protocol for management of the infant of the diabetic mother. ${ }^{3}$

\section{Results}

A total of 25 babies were treated during the study period of whom nine were preterm (range 33-40 weeks' gestation). Mean birth weight was $1872 \mathrm{~g}$ (range 730-2300). Seven mothers had preeclampsia, three essential hypertension, one systemic lupus erythematosis, and one cryptogenic chronic renal failure. Three were receiving $\beta$ blockers (metoprolol or atenolol). Irritability was present in seven infants. Mean age at starting glucagon was 15.7 hours (range 3-41).

In 23 cases the blood glucose concentration rose above $4 \mathrm{mmol} / \mathrm{l}$ on the initial dosage of $0.5 \mathrm{mg} /$ day, in 20 within three hours (see figure). Maximum daily infusion rate of glucagon was $0.5 \mathrm{mg}$ in 16 infants, $1 \mathrm{mg}$ in five, and greater in four, to a maximum of $20 \mathrm{mg} /$ day in one case. Mean duration of treatment was 81 hours (range six hours to three weeks). Five infants developed hyperglycaemia (a glucose concentration $>10 \mathrm{mmol} / \mathrm{l}$ ), which responded to reduction of the infusion rate. Hypoglycaemia recurred in three infants after interruption of the infusion, and in a further six when the glucagon dosage was rapidly decreased after an adequate initial response. In only three of the latter was it possible to restore blood glucose concentrations by reintroducing glucagon. In the other three, hydrocortisone was required. Steroids were given in a further two cases (at 30 minutes and six hours respectively after

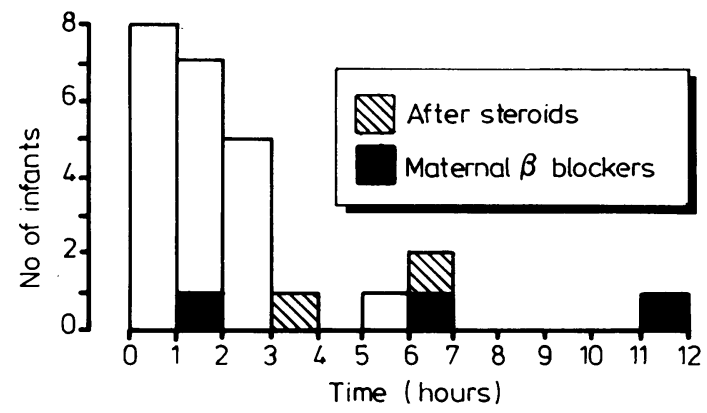

Figure Time until blood glucose concentration was $\geqslant 4 \mathrm{mmol} / \mathrm{l}$. 
commencing glucagon) because of failure of initial response and in three cases of partial response with prolonged marginal hypoglycaemia, at 11 hours, 70 hours, and nine days. Hyperinsulinism lasting six weeks was found in the infant who received steroids after 30 minutes. Of the three infants whose mothers received $\beta$ blockers, two showed delayed response to glucagon at seven and 12 hours respectively, although the third infant responded within two hours (figure).

\section{OUTCOME}

Two infants died from unrelated causes. Necrotising enterocolitis developed during treatment in one infant who had been badly asphyxiated and whose mother was treated with metoprolol. In this case hypoglycaemia was refractory and despite $20 \mathrm{mg} /$ day of glucagon, intravenous hyperalimentation, and corticosteroid the blood glucose concentration was barely maintained at $2 \mathrm{mmol} / \mathrm{l}$. Low thyroxine and cortisol concentrations were found; these eventually resolved spontaneously. There was one case of rectal bleeding with proctoscopic appearances of cows' milk protein intolerance, and another of mild diarrhoea. Two infants became transiently thrombocytopenic and in one of these there was unexplained hyponatraemia. Of the four infants who developed convulsions, two had been badly asphyxiated and one had suffered intracranial haemorrhage. In only one case might hypoglycaemia have been responsible for the convulsions.

At follow up 20 infants are normal and three developmentally delayed. Of the abnormal children, however, one has congenital rubella syndrome and one suffered intracranial haemorrhage. The third is the child described above, with refractory hypoglycaemia. Four otherwise normal children have squints.

\section{Discussion}

The causes of hypoglycaemia in infants who are small for gestational age remain uncertain. Reduction in hepatic glycogen stores is one factor, but reduced gluconeogenesis due to delayed maturation of phosphoenol pyruvate carboxykinase may also be important. ${ }^{4}$ Although an occasional infant may show relatively low glucagon concentrations, ${ }^{2}$ Mestyan et al have shown a degree of resistance to the gluconeogenic effects of glucagon in hypoglycaemic infants who were small for gestational age,, suggesting that supraphysiological concentrations may be necessary in these babies.

We have used a continuous infusion of glucagon, in a dosage similar to that shown by Mestyan et al to effect a rise, albeit blunted, in blood glucose in hypoglycaemic infants who were small for gestational age $(0.2 \mu \mathrm{g} / \mathrm{kg} / \mathrm{min})$. As $28 \%$ of our subjects required an increase in glucagon infusion to above $0.5 \mathrm{mg} /$ day, it is likely that this is near the minimum effective dose. We believe it to be more physiological than the use of a single bolus of $0.2 \mathrm{mg} / \mathrm{kg}$ as advocated by Mehta et al as, in adults and older children, bolus doses of only $1 \mathrm{mg}$ are often associated with vomiting. Mehta et al report a sustained glycaemic effect in a small group of term infants treated with a single bolus. Unfortunately it is not possible to compare these studies as his group of larger babies with low glucagon concentrations may well represent a different population from our own group of low birthweight infants who were small for gestational age, and the course of the only infant weighing less than $2500 \mathrm{~g}$ is not fully documented. Further studies in infants of low birth weight would be of value.

The poor response in infants born to mothers receiving $\beta$ blockers suggests that these babies may be particularly resistant to the effect of glucagon. Glucagon releases catecholamines from the adrenal medulla, ${ }^{6}$ and this may be an important aspect of its therapeutic action.

Our experience with a heterogeneous group of patients shows that intravenous glucagon is effective in the infant who is small for gestational age, in that adequate blood glucose concentrations were obtained in $80 \%$ of babies within three hours of starting treatment. We suggest that in those infants who respond, glucagon be discontinued slowly (over 24 hours or more), and that infusions be rapidly resited during treatment, as rebound hypoglycaemia may be resistant to treatment. If response does not occur within four hours we recommend the early addition of hydrocortisone as late response is uncommon. Similarly, if there is no response to $2 \mathrm{mg} /$ day, further dosage increase is unlikely to be of value. Resistant hypoglycaemia is particularly likely after $\beta$ blockade.

We believe that glucagon is a safe and effective treatment for hypoglycaemia in the infant who is small for gestational age, enabling early introduction of oral feeds and avoiding the need for highly concentrated dextrose infusions.

\footnotetext{
References

I Aynsley-Green A, Soltiez G. Disorders of blood homeostasis in the neonate. In: Roberton NRC, ed. Textbook of neonatology. London: Churchill Livingstone, 1986:611-2.

2 Mehta A, Wootton R, Cheng KN, Penfold P, Halliday D, Stacey TE. Effect of diazoxide or glucagon on hepatic glucose production rate during extreme neonatal hypoglycaemia. Arch Dis Child 1987;62:924-30.

${ }^{3}$ Lloyd DJ, Duffty P. Management of the infant of the diabetic
} 
mother; recent experience. In: Sutherland HW, Stowers JM, eds. Carbohydrate metabolism in pregnancy and the newborn. Edinburgh: Churchill Livingstone, 1984:144-9.

4 Mestyan J, Saltes FG, Schultz K, Howarth M. Hyperaminoacidaemia due to the accumulation of gluconeogenic amino acid precursors in hypoglycaemic small for gestational age infants. $J$ Pediatr 1975;87:409-14.

${ }^{5}$ Mestyan J, Schultz K, Soltesz G, Howarth M. The metabolic effects of glucagon infusion in normoglycaemic and hypogly- caemic small for gestational age infants II. Changes in plasma amino acids. Acta Paediatr Acad Sci Hung 1976;17:245-53.

${ }^{6}$ Bloom SR. Glucagon. Br J Hosp Med 1975;13:150-8.

Correspondence and requests for reprints to Dr P E Carter, Royal Aberdeen Children's Hospital, Cornhill Road, Aberdeen AB9 2ZA.

Accepted 5 April 1988

\title{
Acute pneumonia in Zimbabwe: bacterial isolates by lung aspiration
}

\author{
M O IKEOGU \\ Department of Paediatrics, Mpilo Central Hospital, Bulawayo, Zimbabwe
}

SUMMARY Forty children, aged 2 months to 11 years, with severe acute pneumonia were investigated by needle aspiration of the lung. Fourteen organisms were isolated in only 13 patients. Streptococcus pneumoniae was isolated in six patients, Staphylococcus aureus in three, and Haemophilus influenzae in two. Two patients had mixed organisms.

Severe acute lower respiratory infection is common in developing countries and is a major cause of mortality. Studies from some developing countries indicate a high prevalence of bacteria in the aetiology of pneumonia in hospitalised children. ${ }^{12}$ This may not be universally the case. The present study was aimed at determining whether bacteria are the dominant cause of pneumonia in children in $\mathrm{Zim}$ babwe as has been found in some developing countries.

\section{Patients and methods}

The study was conducted between February and August 1987. All children admitted under one of the three paediatric firms with severe pneumonia were investigated by needle aspiration if they had not received any antibiotics before admission. The needle aspiration was performed according to a standard method with slight modification. ${ }^{3}$ The aspirate was cultured on blood and chocolate agar, and tryptose phosphate or brain-heart infusion broth. The positive growths were indentified by standard bacteriological methods. Initially negative cultures were subcultured for 10 days.

In the period under consideration 153 children were discharged with a final diagnosis of either lobar pneumonia or bronchopneumonia. Of these 40 qualified for lung aspiration and had the procedure carried out. All were between the ages of 2 months and 11 years. All had a history of respiratory infection of less than five days' duration. The extent of the consolidation was determined by physical examination and confirmed on a chest radiograph and involved at least a whole lobe.

\section{Results}

Fourteen bacterial organisms were isolated by lung aspiration from 13 children amounting to a positive isolation rate of $32.5 \%$. Of the single isolates the dominant organism was Streptococcus pneumoniae, which was isolated in six patients, followed by Staphylococcus aureus in three, and Haemophilus influenzae in two. Two patients had mixed organisms, one with $S$ pneumoniae and $H$ influenzae and another with $S$ aureus and Mycobacterium tuberculosis. The $M$ tuberculosis was isolated from sputum culture.

There were no deaths attributable to lung aspiration. The only death in the series was an 18 month old child with severe kwashiokor and right upper lobe pneumonia due to $H$ influenzae and large intracerebral abscess presumably due to the same organism. One patient had slight haemoptysis that cleared within 30 minutes, and another had a small pneumothorax that cleared spontaneously.

\section{Discussion}

Needle aspiration of the lung is the only definitive way of establishing the bacterial organism responsible for acute pneumonia. Studies from developing countries using this technique have established beyond doubt the dominant role of bacteria in the aetiology of acute pneumonia in hospitalised children. ${ }^{124}$ This, together with widespread mal- 